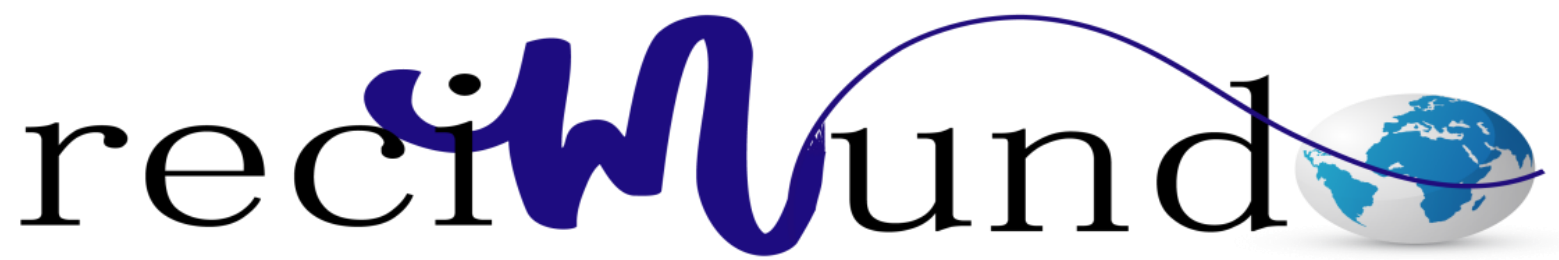

Revista Científica Mundo de la Investigación y el Conocimiento

Eduardo Vera Muthre ${ }^{\mathrm{a}}$; Carmen Lazo Caicedo ${ }^{\mathrm{b}}$; Sonia Cedeño Loor ${ }^{\mathrm{c}}$; Cesar Bravo Bermeo $^{\mathrm{d}}$

Actualización sobre el cáncer de tiroides

Update on thyroid cancer

Revista Científica Mundo de la Investigación y el Conocimiento. Vol. 2 núm.3, julio, ISSN: 2588-073X, 2018, pp. 16-42

DOI: 10.26820/recimundo/2.(3).julio.2018.16-42

Editorial Saberes del Conocimiento

Recibido: 05/04/2018

Aceptado: 15/06/2018

Publicado: 30/07/2018

Correspondencia: eduardo.veram@ug.edu.ec

a. Docente Carrera de Medicina. Universidad de Guayaquil; eduardo.veram@ug.edu.ec

b. Docente Carrera de Medicina. Universidad de Guayaquil; carmen.1azoc@ug.edu.ec

c. Docente Carrera de Medicina. Universidad de Guayaquil; leylla.cedenol@ug.edu.ec

d. Docente Carrera de Medicina. Universidad de Guayaquil; cesar.bravob@ug.edu.ec 


\section{RESUMEN}

El cáncer de tiroides es frecuente en el Ecuador, con mayor prevalencia en mujeres, observando una buena respuesta al tratamiento quirúrgico y al yodo radioactivo, tiene por lo general buen pronóstico. La principal manifestación clínica de los pacientes que asisten a la consulta médica es la aparición de un nódulo tiroideo. El diagnostico se realiza mediante exámenes de imagen como la ecografía, laboratorio, pruebas de función tiroidea, punción aspiración con aguja fina (PAAF) y biopsia que es el procedimiento que establece el tipo de lesión histológica de la glándula.

Palabras claves: Cáncer de tiroides, Neoplasia, Aspiración con aguja con aguja fina, Biopsia. 


\title{
Actualización sobre el cáncer de tiroides
}

Vol. 2, núm. 3., (2018)

Eduardo Vera Muthre; Carmen Lazo Caicedo; Sonia Cedeño Loor; Cesar Bravo Bermeo

\begin{abstract}
Thyroid cancer is frequent in Ecuador, with a higher prevalence in women, it usually has a good prognosis, observing a good response to surgical treatment, and radioactive iodine. The main clinical manifestation of the patients who attend the medical consultation is the appearance of a thyroid nodule. The diagnosis is made through imaging tests such as ultrasound, laboratory, thyroid function tests and fine needle aspiration (FNA) and biopsy, which is the procedure that establishes the type of histological injury of the gland.
\end{abstract}

Keywords: Thyroid cancer, Neoplasia, Needle aspiration with fine needle, Biopsy. 
Eduardo Vera Muthre; Carmen Lazo Caicedo; Sonia Cedeño Loor; Cesar Bravo Bermeo

\section{Introducción.}

El cáncer de tiroides se encuentra actualmente como la neoplasia maligna más común del sistema endocrino. En Latinoamérica ésta patología aparece en 9 de cada 100.000 personas por año, afecta en mayor grado a las mujeres aunque con baja tasa de mortalidad. Ecuador, Brasil, Costa Rica y Colombia presentan las tasas más elevadas de la región ${ }^{1}$

En la población femenina se ha reportado incremento del número de nuevos casos, obteniendo las siguientes cifras, en Ecuador un 0,016\% por cada 100.000 habitantes, Brasil $(0,014 \%)$, Costa Rica $(0,012 \%)$ y Colombia $(0,011 \%)$, valores semejantes a los de países desarrollados; y las cifras de mortalidad representan en Ecuador y México un 0,0009\% por cada 100.000 habitantes, Colombia $(0,0008 \%)$, Perú $(0,0007 \%)$ y Panamá $(0,0005 \%)^{2}$.

En Ecuador, según la Sociedad de Lucha contra el Cáncer (SOLCA), las ciudades con más incidencia de casos son Quito, Cuenca, Loja, Guayaquil, las ciudades de las provincias de Manabí y el Oro les siguen en frecuencia”3. El cáncer diferenciado de tiroides (DTC) que incluye al carcinoma papilar y folicular, comprende más del $90 \%$ de este tipo de cáncer ${ }^{4}$.

Clínicamente la manifestación inicial del Ca de tiroides es la presencia de un nódulo tifoideo pero solo entre el 5 y $7 \%$ es de origen maligno ${ }^{5}$, también suele presentarse acompañado de una linfadenopatia, entre otros síntomas se encuentran el dolor a nivel del cuello, ronquera o cambios en la voz, disfagia, disnea y tos sin causa aparente ${ }^{6}$.

Para el diagnóstico es esencial una historia clínica y examen físico completo, los principales métodos de diagnóstico usados son la ecografía tiroidea, pruebas de función tiroidea 


\section{Actualización sobre el cáncer de tiroides}

Vol. 2, núm. 3., (2018)

Eduardo Vera Muthre; Carmen Lazo Caicedo; Sonia Cedeño Loor; Cesar Bravo Bermeo

(T3, T4, TSH), finalmente la punción por aspiración con aguja fina (PAAF), procedimiento con el que se arriba a un diagnóstico de certeza ${ }^{7}$.

Dentro del protocolo de tratamiento del cáncer tiroideo se encuentra la administración de yodo radiactivo, terapia con hormona tiroidea, radioterapia, y la cirugía que es el tratamiento definitivo, cuyo abordaje dependerá de la estadificación de la neoplasia ${ }^{8}$.

Debido a la elevada frecuencia del $\mathrm{Ca}$ de tiroides en la población femenina a nivel mundial que se refleja en nuestra localidad, y por no tener suficiente información se presenta como una necesidad hacer una revisión del Ca de tiroides para poner al día los nuevos enfoques y actualización médico quirúrgica de esta patología endocrinológica que sirva como fundamento teórico para el abordaje clínico, diagnóstico y terapéutico de los pacientes que presentan esta enfermedad neoplásica maligna de la glándula tiroidea

Si bien es cierto que, las enfermedades de la glándula tiroides requieren de la atención de especialistas en endocrinología, la evaluación inicial queda en manos de los médicos generales o de especialistas en Atención Primaria de Salud, los que en base al cuadro clínico elaboran un diagnostico presuncional y refieren a los pacientes al 2do o 3er nivel de atención. Hay que señalar que en el caso de las neoplasias de la glándula tiroides la presunción diagnostica no es difícil porque habitualmente su presentación inicial es de una tumoración observable o palpable en el territorio de la glándula, referida en la literatura médica como nódulo tiroideo. Con este diagnóstico presuntivo la referencia debe ser inmediata para que el paciente sea sometido oportunamente a una punción biopsia (PAFF) cuyo estudio determina si se trata de neoplasia benigna o maligna. 
Eduardo Vera Muthre; Carmen Lazo Caicedo; Sonia Cedeño Loor; Cesar Bravo Bermeo

Una vez establecido el diagnóstico y tipo de Ca de tiroides los estudios de imagen y de laboratorio determinan el plan terapéutico a seguir, esto es, la aplicación de quimioterapia, radioterapia, cirugía, radioactividad y seguimiento. Este trayecto con el cuadro clínico previo al diagnóstico, el establecimiento de un diagnóstico de certeza, el tratamiento, la determinación de complicaciones y/o recurrencias, constituyen la evolución de la enfermedad.

Este articulo tiene el propósito, contribuir a la preparación y formación continua de los estudiantes de medicina, internos y médicos generales de APS, quienes, no pocas veces, por considerar que el Cáncer de Tiroides cae en el ámbito de la endocrinología se la observa de forma superficial desconociendo la complejidad de la enfermedad que amenaza la vida y requiere para su solución participación medica multidisciplinaria.

\section{Método.}

Se trata de una revisión documental en la que se registra literatura mundial de los 5 últimos años sobre el cáncer de tiroides con un análisis previo sobre el desarrollo normal de la glándula pasando por la embriología, anatomía, histología, patología, función, presentación clínica, métodos de diagnóstico, tratamiento con yodo radioactivo y cirugía, complicaciones, resultados y pronóstico.

\section{Tiroides}

\section{Embriología}

El desarrollo de la tiroides empieza durante la tercera y cuarta semana de gestación, formándose el agujero ciego que sale como un divertículo desde el suelo de la faringe, esta 


\section{Actualización sobre el cáncer de tiroides}

Vol. 2, núm. 3., (2018)

Eduardo Vera Muthre; Carmen Lazo Caicedo; Sonia Cedeño Loor; Cesar Bravo Bermeo

estructura proviene de una proliferación endodérmica. Así mismo continúa con el desarrollo del

conducto tirogloso a través de un conjunto solido de células que se extienden en el interior tubular hasta alcanzar el hueso hioides. Alrededor de la séptima semana ocupara su posición definitiva, a nivel del tercer y sexto anillo traqueal.

El conducto tubular se separa en diversos cordones celulares para la formación del istmo y los lóbulos laterales del tiroides. El conducto tirogloso durante la octava semana se degenera y el extremo superior forma parte del agujero ciego, en la mitad de los casos, en el extremo inferior, permanece como el lóbulo piramidal localizada generalmente en el lado izquierdo de la tiroides 9 .

\section{Anatomía}

La glándula tiroides es parte del sistema endocrino, tiene un peso aproximado de 30 gramos y además un flujo sanguíneo de $5 \mathrm{ml} / \mathrm{gr}$ por minuto. Su estructura es bilobulada, se sitúa anterior y lateral a los cartílagos tiroides, justo en la unión de la laringe y tráquea ${ }^{10}$

La tiroides consta de dos lóbulos laterales unidos por el centro a través de un istmo, ubicado en situación anterior, al nivel o inmediatamente por debajo del cartílago cricoides. El tubérculo de Zuckerkandl es una extensión del tejido tiroideo posterior de los lóbulos laterales, es importante en la tiroidectomía total, debido a su gran relación con el nervio laríngeo recurrente ${ }^{11}$.

La tiroides tiene dos sistemas de irrigación uno superior y otro inferior, con múltiples anastomosis $^{12}$, entre ellas se encuentran: 
Eduardo Vera Muthre; Carmen Lazo Caicedo; Sonia Cedeño Loor; Cesar Bravo Bermeo

Tiroidea superior: es rama de la arteria carótida externa, desciende hasta el extremo superior del nervio laríngeo superior y se bifurca dando lugar a; una rama anterior, que se comunica con su homóloga contralateral y otra posterior la cual se une con ramas de la arteria tiroidea inferior

Tiroidea inferior: proviene de la arteria subclavia, recorre por delante y por detrás al nervio laríngeo recurrente y se ramifica en dos, una superior que se une con la rama posterior de la arteria tiroidea superior, y la rama inferior encargada de brindar irrigación a la región inferior de la glándula, en algunos casos está ausente.

Las siguientes venas se encargan del drenaje:

Vena tiroidea superior: desemboca en la vena yugular interna.

Vena tiroidea media: desemboca de forma similar a la superior.

Vena tiroidea inferior: desemboca en las venas braquiocefálicas

Las principales estructuras nerviosas en relación a la glándula son:

Nervio laríngeo superior: pasa paralelo a los vasos sanguíneos tiroideos superiores, y su función es inervar el músculo cricotirioideo, su contracción dilata las cuerdas vocales y su lesión produce trastornos temporales y leves como la hipofonía.

Nervio laríngeo recurrente inferior: el cual inerva todos los músculos laríngeos exceptuando el cricotirioideo, su contusión unilateral involucra parálisis en las cuerdas vocales produciendo la voz bitonal y su lesión bilateral, provoca afonía y dificultad respiratoria. 


\section{Actualización sobre el cáncer de tiroides}

Vol. 2, núm. 3., (2018)

Eduardo Vera Muthre; Carmen Lazo Caicedo; Sonia Cedeño Loor; Cesar Bravo Bermeo

\section{Histología}

La tiroides está integrada por dos tipologías de células:

Células foliculares: Producen las hormonas tiroideas, triyodotironina (T3) y tiroxina (T4). Estas hormonas regulan el metabolismo, si hay demasiada producción logran ocasionar hipertiroidismo y si es escasa hipotiroidismo. La secreción de hormona tiroidea es regulada por la hormona tirotropina (TSH) producida en la glándula pituitaria.

Células C o parafoliculares: Estas células producen calcitonina, hormona que regula el metabolismo del calcio. Se confinan alrededor de los folículos. Cada ejemplar de célula genera un tipo de cáncer diferente y de esto depende la gravedad de la enfermedad y el procedimiento que se deberá realizar.

Clínicamente el $\mathrm{Ca}$ de tiroides se manifiesta con la aparición de un nódulo solitario evidente o palpable, que puede estar relacionado o no con síntomas de hipotiroidismo y en un $13 \%$ con hipertiroidismo, inicialmente gran parte de estos casos son asintomáticos y conforme avanza la patología, se presenta el crecimiento del nódulo, alteraciones en la voz, disfonía, disfagia o hemoptisis. Fisiología

La secreción de las hormonas tiroideas tiroxina (T4) y triyodotironina (T3) se encuentra sujeta a la estimulación de la tirotropina hipofisaria (TSH). La producción de hormona tiroidea (HT) obedece especialmente de la reserva de yodo exógeno. El equilibrio del yodo se conserva 
Eduardo Vera Muthre; Carmen Lazo Caicedo; Sonia Cedeño Loor; Cesar Bravo Bermeo

por los aportes dietéticos y por la absorción de medicamentos, contrastes consumidos como métodos diagnósticos o suplementos alimenticios ${ }^{13}$

Las hormonas tiroideas poseen receptores en la mayoría de tejidos, y regulan casi todas las funciones del metabolismo intermedio. Las hormonas $\mathrm{T} 3 \mathrm{y} \mathrm{T} 4$, tienen un amplio efecto sobre el metabolismo y desarrollo. El déficit de hormona tiroidea produce destacados efectos durante la maduración fetal y también en los primeros meses de vida ${ }^{14}$.

En el niño, las principales alteraciones son el deficiente desarrollo intelectual y el retardo del crecimiento. El déficit intelectual, está relacionado al tiempo que persiste la falta de aporte hormonal y es irreversible; el retraso en el crecimiento tiene origen estrictamente de tipo metabólico, y en estos casos el crecimiento se acomoda ágilmente a su ritmo normal después de establecer el tratamiento. En el adulto, su manifestación principal está relacionada con alteraciones metabólicas. Esta consecuencia contiene cambios en el consumo de oxígeno y también influye en la desintegración de proteínas, carbohidratos, grasas y vitaminas ${ }^{15}$.

A continuación se menciona las acciones más importantes de las hormonas tiroideas:

Son especialmente necesarias para el crecimiento y desarrollo durante la infancia.

Tienen acción calorígena y termorreguladora

Aumentan la utilización de oxígeno.

Estimulan la síntesis y degradación de las proteínas.

Regulan las mucoproteínas y el agua extracelular. 


\section{Actualización sobre el cáncer de tiroides}

Vol. 2, núm. 3., (2018)

Eduardo Vera Muthre; Carmen Lazo Caicedo; Sonia Cedeño Loor; Cesar Bravo Bermeo

Participan en la síntesis y degradación de las grasas.

Median la síntesis de glucógeno y la utilización de glucosa.

Son útiles en la formación de vitamina $\mathrm{A}$, por medio de los carotenos.

Son indispensables para el desarrollo adecuado del sistema nervioso central y periférico.

Actúan en los mecanismos de contracción muscular y motilidad intestinal.

\section{Nódulos Tiroideos}

Las alteraciones de tamaño y forma de la glándula tiroides generalmente pueden ser detectadas por los pacientes.

Cuando toda la glándula está incrementada de tamaño, son denominados bocios difusos, en cambio sí se encuentra aumentada de volumen y tiene uno o más nódulos, se denominan a estos casos bocios nodulares. En general un bocio no siempre implica que estemos frente a un cáncer tiroideo, ya que muchas veces los bocios difusos y nodulares son ocasionados por un desequilibrio en ciertas hormonas ${ }^{16}$.

Los nódulos tiroideos pueden aparecer a cualquier edad, pero éstos se presentan más menudeo en adultos de mediana edad. En muchos pacientes que presentan nódulos pequeños es necesario realizar una ecografía, y habitualmente este tamaño indica una patología benigna, como por ejemplo quistes con abundante líquido u hormonas tiroideas, son conocidos como coloides. 


\section{Actualización sobre el cáncer de tiroides}

Vol. 2, núm. 3., (2018)

Eduardo Vera Muthre; Carmen Lazo Caicedo; Sonia Cedeño Loor; Cesar Bravo Bermeo

Aquellos nódulos solidos o con poco líquido en su interior tienen mayor probabilidad de malignidad que los nódulos llenos de líquido. A pesar de esto no todos son cáncer, porque existen algunos casos como los adenomas y nódulos hiperplásicos que poseen abundantes células sin ser cancerosas ${ }^{17}$.

Algunas veces los nódulos benignos no requieren tratamiento, siempre y cuando mantengan el mismo tamaño y no produzcan síntomas, puede que otros requieran algún tipo de tratamiento.

\section{Cáncer de Tiroides}

\section{Epidemiologia}

El Ca de tiroides se encuentra actualmente como la neoplasia maligna más común del Sistema Endocrino. En Latinoamérica, Ecuador, Brasil, Costa Rica y Colombia tienen las tasas más elevadas de esta patología que se detecta en 9 de cada 100.000 personas por año, afectando principalmente a la población femenina, con una baja tasa de mortalidad y un pronóstico favorable. Cerca de 26.000 casos nuevos de cáncer de tiroides se diagnosticaron en América Central y Suramérica en el 2012, el $82 \%$ de ellos fue en mujeres, según el reporte los Centros de Control y Prevención de Enfermedades (CDC) de EE.UU.

Según la Sociedad Española de Oncología Médica (SEOM) informó en el 2014 que, el Ca de tiroides es una neoplasia rara, constituye menos del $1 \%$ de los tumores malignos, aparece entre de 2 y 20 casos por 100.000 habitantes y año ${ }^{18}$. 


\section{Actualización sobre el cáncer de tiroides}

Vol. 2, núm. 3., (2018)

Eduardo Vera Muthre; Carmen Lazo Caicedo; Sonia Cedeño Loor; Cesar Bravo Bermeo

Esta patología representa cerca del $90 \%$ de todas las neoplasias endocrinas, y es la principal causa de muertes por encima de los demás tumores malignos de este sistema. La ocurrencia de esta entidad ha incrementado durante los últimos años pero las tasas de mortalidad se han mantenido constantes. La incidencia se modifica según la situación geográfica, en aquellas zonas con déficit alimentario de yodo es más frecuente la aparición de estos carcinomas.

\section{Factores de riesgo}

No se encuentran bien establecidas las causas de esta patología; por lo general en los pacientes con $\mathrm{Ca}$ de tiroides no se conocen factores de riesgos o antecedentes en sus registros médicos. No obstante, existen diversas situaciones en las que se ha percibido una frecuencia más elevada.

A continuación citamos los factores de riesgo más importantes:

Exposición a radiaciones ionizantes: las personas que han tenido exposición a radiación en la infancia tiene mayor probabilidad de presentar cáncer de tiroides por eso este factor de riesgo es el más reconocido.

Aquellos sujetos que durante la infancia fueron expuestos a radiación, ya sea con fines terapéuticos como radioterapia de cabeza y cuello, o por proximidad a fuentes radiactivas en el medio ambiente, por ejemplo accidentes nucleares, tienen mayor riesgo de padecer cáncer de tiroides, hasta $50 \%$ más que el resto de la población ${ }^{19}$.

Factores genéticos: el carcinoma medular, tiene una presentación de tipo familiar en el 25 $\%$ de los casos, sin embargo, este es el tipo menos frecuente de Ca de tiroides. En el síndrome de 
Eduardo Vera Muthre; Carmen Lazo Caicedo; Sonia Cedeño Loor; Cesar Bravo Bermeo

Gardner o la enfermedad de Cowden, el carcinoma papilar puede aparecer como una manifestación de estas enfermedades hereditarias.

Dieta deficiente en yodo: guarda especial relación con los carcinomas papilares y foliculares.

Sexo y edad: son más habituales en pacientes de sexo femenino y en las edades comprendidas entre la tercera y quinta década de la vida. Parece relacionarse una mayor ocurrencia de este tipo de cáncer en mujeres con historia reproductiva y el uso de anticonceptivos orales ${ }^{20}$.

Tipos de cáncer de tiroides

Dentro de los principales tipos se encuentran:

Tumores de Células foliculares

Diferenciados: Carcinoma Papilar, Folicular y de células Hürthle

Indiferenciado: Anaplásico, Tumores de células C

Carcinoma medular

En su mayoría se presentan como cáncer de tiroides diferenciados ${ }^{21}$.

Carcinoma Papilar.

Conocido también como adenocarcinoma papilar, es el tumor maligno más habitual de la glándula tiroides. Estos se caracterizan por un lento crecimiento y suelen afectar a un único 


\section{Actualización sobre el cáncer de tiroides}

Vol. 2, núm. 3., (2018)

Eduardo Vera Muthre; Carmen Lazo Caicedo; Sonia Cedeño Loor; Cesar Bravo Bermeo

lóbulo de esta glándula. Este cáncer frecuentemente se propaga hacia los ganglios linfáticos del cuello, sin embargo con el tratamiento apropiado es de buen pronóstico causando raras veces muerte del paciente.

A su vez este consta de varios subtipos entre los cuales se encuentran:

Folicular o variante folicular-papilar mixta siendo el que aparece con mayor frecuencia.

Carcinoma papilar (células cilíndricas, células altas y esclerosante difuso e insular), estos son menos comunes ${ }^{21}$.

\section{Carcinoma Folicular}

Este tiene una mayor incidencia en países con un insuficiente aporte de yodo en sus dietas. Es menos frecuente que se propaguen a los ganglios linfáticos, aun así pueden diseminarse en otras regiones del cuerpo, como huesos y pulmones.

\section{Cáncer de células Hürthle.}

También denominado como carcinoma de células oxífilas. Es la más infrecuente de estas neoplasias, por este motivo su diagnóstico y tratamiento es más complicado.

\section{Carcinoma Medular.}

Se presenta en un $4 \%$ de los cánceres tiroideos, su desarrollo es a partir de la células C productoras de calcitonina. Antes de que se detecte la aparición de un nódulo tiroideo puede llegar a propagarse hacia los ganglios linfáticos, pulmones o al hígado.

Su diagnóstico y tratamiento son complejos, Existen dos tipos: 
Eduardo Vera Muthre; Carmen Lazo Caicedo; Sonia Cedeño Loor; Cesar Bravo Bermeo

Carcinoma Medular Esporádico representa gran parte de este tipo de cánceres, no es hereditario, afecta principalmente a los adultos en edades avanzadas y únicamente a un lóbulo tiroideo.

Carcinoma Medular Familiar aparece en un menor número de casos de esta patología y si es hereditaria, la mayoría de veces se originan en los niños y adultos jóvenes, y afectan varias zonas de ambos lóbulos tiroideos.

\section{Cáncer Anaplásico}

Conocido también como carcinoma indiferenciado, es una de las formas de presentación más raras de esta neoplasia. Se tiene la creencia de que en algunas ocasiones su desarrollo es a partir de un previo carcinoma papilar o folicular.

Su denominación como indiferenciado es determinada porque las células guardan muy poca o casi nula relación con las células normales de la glándula tiroidea. Su forma de propagación es rápida afectando diferentes partes del cuerpo, teniendo un difícil manejo.

\section{Cánceres menos frecuentes de la Tiroides}

Representan en su conjunto aproximadamente un $4 \%$ de los cánceres tiroideos entre ellos están los linfomas de tiroides, sarcomas y varios más infrecuentes aún.

\section{Cuadro clínico.}

La primera manifestación clínica se caracteriza por la aparición de una masa en el cuello que tiende a crecer muy rápido, se acompaña de dolor en la región anterior que puede irradiarse a 


\section{Actualización sobre el cáncer de tiroides}

Vol. 2, núm. 3., (2018)

Eduardo Vera Muthre; Carmen Lazo Caicedo; Sonia Cedeño Loor; Cesar Bravo Bermeo

los oídos, también pueden aparecer alteraciones en la voz del paciente o ronquera sin alguna otra causa aparente, puede aparecer también disfagia y odinofagia).

La dificulta respiratoria y tos persistente que no son originados por procesos gripales o infecciosos de las vías respiratorias, aparecen en los casos más graves.

\section{Diagnostico.}

Se debe realizar una exhaustiva historia clínica y exploración física, en la cual se implemente el uso de un laringoscopio. Dentro de las técnicas para un diagnóstico precoz, contamos con la ecografía y la punción aspiración con aguja fina (PAAF).

La ecografía ayuda a evidenciar una mala delimitación del nódulo, de forma irregular por su hipoecogeneidad, ausencia de halo de seguridad, calcificaciones, una comparación del crecimiento con exámenes previos y vascularización intranodular ${ }^{22}$.

La PAAF tiene una sensibilidad y especificidad del 90\%; se lo efectúa directamente cuando el nódulo se puede palpar, caso contrario el uso de la ecografía nos ayudara de guía, también es utilizada cuando una muestra anterior resulto insuficiente.

A través de la PAFF clasificamos los nódulos como benignos, indeterminados o malignos; dependiendo del resultado citológico obtenido determinaremos nuestro actuar médico.

Para clasificar las citologías tiroideas disponemos del Sistema Bethesda ${ }^{23}$, el cual nos da 6 categorías diagnósticas, cada una representa un porcentaje distinto de riesgo para malignidad, por lo tanto, también cuentan con protocolos de tratamiento diferente. Este sistema fue empleado con el objetivo de disminuir la cantidad de cirugías innecesarias, obteniendo con ello una 
disminución de aspirados indeterminados, un alza de los aspirados benignos, y sin aumentar lo malignos.

\section{Tratamiento Quirúrgico}

La resección quirúrgica de estos tumores es el tratamiento de elección, se emplean diversas técnicas quirúrgicas:

\section{Hemitiroidectomía o lobectomía tiroidea}

Algunos autores defienden esta técnica, que consiste en la resección únicamente del lóbulo afectado, en grupos de paciente con bajo riesgo. Las ventajas que posee este procedimiento debido a la conservación de tejido tiroideo funcional, es evitar la terapia sustitutiva hormonal y disminuir el riesgo de complicaciones en el lóbulo contralateral. Las desventajas que posee son un aumento en el porcentaje de recidivas y retraso en el diagnóstico, dado que se dificulta el rastreo corporal total con yodo radioactivo ${ }^{24}$.

\section{Tiroidectomía casi-total}

Esta técnica consiste en la extirpación de toda la glándula a excepción de una parte glandular posterior, con el objetivo de evitar lesiones en las glándulas paratiroides y nervio laríngeo recurrente. Este remanente se elimina con Yodo radioactivo (I131), al igual que cualquier resto de tejido funcional existente ${ }^{25}$. 


\section{Actualización sobre el cáncer de tiroides}

Vol. 2, núm. 3., (2018)

Eduardo Vera Muthre; Carmen Lazo Caicedo; Sonia Cedeño Loor; Cesar Bravo Bermeo

\section{Tiroidectomía total}

Es la técnica más utilizada para el tratamiento de carcinoma papilar, resecando por completo a la glándula. Posteriormente se consigue una ablación completa con el uso de I131 que elimina el tejido funcionante oculto y se facilita también el seguimiento con medicina nuclear y tiroglobulina. A pesar de que esto conlleve a un mayor número de complicaciones, este procedimiento presenta tasas muy bajas de recurrencia y un mayor porcentaje de supervivencia. La mayor desventaja que posee es el uso de por vida de terapia hormonal sustitutiva.

Es bastante frecuente la diseminación de esta neoplasia en el cuello. La metástasis ganglio se maneja dependiendo de su distribución. Si los ganglios afectados se encuentran en el compartimiento central del cuello, se procederá a realizar un vaciamiento selectivo de esta área V1 en conjunto con la Tiroidectomía. Si los ganglios afectados son parte de la cadena yugulocarotidea, se procederá a la resección selectiva de las áreas 1l, lll y lV.

No está recomendado el vaciamiento profiláctico, ni el conocido “picoteo ganglionar", a no ser que se encuentren adenopatías metastásicas.

El tratamiento de las recidivas consiste en cirugía y yodo radioactivo si el tumor es captaste. La radioterapia se la puede utilizar con un coadyuvante, mientras que la quimioterapia es para uso paliativo.

Tratamiento no quirúrgico

Yodo radioactivo 
Eduardo Vera Muthre; Carmen Lazo Caicedo; Sonia Cedeño Loor; Cesar Bravo Bermeo

Después de que haya sido tratado el tumor primario, se realizará un rastreo con yodo radioactivo previo al haberse suspendido la terapia hormonal sustitutiva. Siempre que el tumor capte yodo, si se reconoce enfermedad local residual o metástasis, se procederá a la administración de I131 en intervalos hasta que ya no se observe alguna captación en la gammagrafía corporal. Mejora significativamente el pronóstico de estos tumores ${ }^{26}$.

\section{Terapia de supresión con Levotiroxina}

Debido a la actividad mitogénica que tiene la FSH sobre la célula tumoral diferenciada, es de gran importancia mantenerla en valores por debajo de los normales, siendo de mayor eficacia en pacientes en estadios $1 \mathrm{l}$ y $\mathrm{lV}$, los cuales mostraron un incremento importante en la supervivencia.

El nivel de supresión aconsejado dependerá del riesgo de recaída. Es así como pacientes con alto riesgo se aconseja un valor de supresión de la TSH entre 0,01 a 0,1 Mui/L, en cambio en pacientes con bajo riesgo los valores aconsejados oscilan entre 0,1 a $0,4 \mathrm{Mui} / \mathrm{L}^{27}$.

\section{Complicaciones de la cirugía de tiroides}

\section{Hemorragia}

La incidencia de hemorragia postquirúrgica es relativamente baja, pero su aparición la hace potencialmente muy grave, pues el hematoma sofocante que provoca puede comprometer en poco tiempo la vía aérea del paciente. La aparición en el postoperatorio inmediato de dolor, distensión cervical y otros síntomas de compromiso aéreo como disnea, estridor o tiraje debe obligarnos a descartar rápidamente la presencia de un hematoma en el área quirúrgica. 


\section{Actualización sobre el cáncer de tiroides}

Vol. 2, núm. 3., (2018)

Eduardo Vera Muthre; Carmen Lazo Caicedo; Sonia Cedeño Loor; Cesar Bravo Bermeo

Infección

Es escasa su frecuencia y puede aparecer de varias formas diferentes, desde celulitis, abscesos superficiales hasta abscesos profundos de cuello. La clínica será de eritema, calor local, dolor, fiebre, leucocitosis, taquicardia. Son ventajosas para el diagnóstico las pruebas de imagen como la ecografía o la TC, así como la realización de una tinción de Gram y un cultivo posterior del exudado.

\section{Hipoparatiroidismo}

Se debe a la exéresis de alguna de las glándulas paratiroides o, muchas veces, a pérdida de la vascularización, traumatismo o hipotermia de la glándula. Se ha propuesto últimamente la implicación de la endotelina-1, un reactante de fase aguda, en la caída de hormona PTH tras la manipulación de las paratiroides. Este descenso de la parathormona provoca una hipocalcemia con hiperfosforemia transitoria o permanente.

Parestesias periorales, tetania, espasmo carpo-pedal, laringoespasmo, convulsiones, cambios de comportamiento, prolongación de QT en ECG, etc. Nos deben alertar y obligar a un tratamiento precoz de esta situación. La hipomagnesemia puede acompañar a este cuadro, presentándose como náuseas y vómitos hasta tetania, convulsiones o arritmias en casos graves.

El diagnóstico se hace, fundamentalmente, con el control postoperatorio de la calcemia total e iónica, los síntomas y signos anteriormente descritos y la presencia de los signos de Chvostek y Trousseau.

Tormenta tirotóxica

Revista Científica Mundo de la Investigación y el Conocimiento. 2 (3). pp. 16-42 
Eduardo Vera Muthre; Carmen Lazo Caicedo; Sonia Cedeño Loor; Cesar Bravo Bermeo

Es extremadamente infrecuente pero potencialmente mortal, por lo que no ha de descuidarse esta situación. Suele afectar a pacientes hipertiroideos y presentarse durante la intervención o en el postoperatorio.

Aparecen signos de estimulación simpática como taquicardia, hipertermia, arritmias. El tratamiento radica en suspender la intervención, bajar la temperatura del paciente y administrar betabloqueantes, propiltiouracilo, corticoides, oxígeno. La prevención debe hacerse preparando bien a este tipo de pacientes previamente a la cirugía.

\section{Lesión del nervio recurrente}

La forma de manifestación de este tipo de complicación es variable. Aparece, la mayoría de las ocasiones, como disfonía, acompañada a veces de disfagia y aspiración, síntomas de parálisis vocal unilateral. En los pocos casos en los que haya sido dañado el nervio recurrente de ambos lados, se presentará como disnea, estridor, tiraje y distress respiratorio inmediatamente posterior a la extubación. La disfonía suele empeorar a medio plazo, aun cuando no se haya seccionado por completo el nervio.

\section{Seguimiento.}

El seguimiento de pacientes calificados libres de enfermedad está encaminado a descubrir prematuramente las eventuales recaídas y poder así brindar tratamientos con limitada morbilidad. La tiroglobulina es utilizado como un marcador tumoral, en aquellos pacientes tratados mediante tiroidectomía total más ablación con yodo ${ }^{27}$. Los niveles de $T g$ habitualmente se hacen indetectables por debajo de $0,5 \mathrm{ng} / \mathrm{Ml}$, a partir del tercer mes, no obstante, algunos pacientes 


\section{Actualización sobre el cáncer de tiroides}

Vol. 2, núm. 3., (2018)

Eduardo Vera Muthre; Carmen Lazo Caicedo; Sonia Cedeño Loor; Cesar Bravo Bermeo

continúan con cifras detectables hasta por dos años. Su elevación debe ser interpretada como recaída, haciendo necesaria la búsqueda de enfermedad macroscópica

Después de dos meses posteriores al tratamiento con cirugía o de la dosis I131 según el caso, se debe hacer control clínico y medición de TSH y Tiroglobulina. Si TSH está apropiadamente suprimida y tiroglobulina baja, control cada 6 meses.

Si no concentran el radioiodo considerar resección quirúrgica o radioterapia. Para realizar el rastreo con 1131 el paciente deberá cambiar la terapia supresiva a triyodotironina por 4 a 6 semanas y quedar sin medicación hormona tiroidea por lo menos 3 semanas antes de la gammagrafía, para permitir una mayor elevación y por menor tiempo del nivel de TSH.

Si hay disponibilidad, se puede administrar TSH recombinante como alternativa. A los seis meses de seguimiento se sugiere realizar una ecografía de cuello, independientemente de los niveles de tiroglobulina, ya que las metástasis a ganglios cervicales consiguen no provocar elevación de la misma ${ }^{26}$. El TAC de tórax está indicado sólo ante la evidencia bioquímica de enfermedad (tiroglobulina elevada o antitiroglobulina (ATG) en ascenso ${ }^{27}$.

\section{Pronóstico}

Habitualmente, estos tumores son de muy buen pronóstico, con una tasa de supervivencia de alrededor del $90 \%$ a los diez años. Son factores de mal pronóstico el sexo masculino, la edad mayor a 45 años, el tamaño del tumor mayor de 1,5 cm, la diferenciación celular pobre, las variedades histológicas desfavorables (difusa esclerosante, células altas, células columnares), y los estadios III y IV. 
Eduardo Vera Muthre; Carmen Lazo Caicedo; Sonia Cedeño Loor; Cesar Bravo Bermeo

\section{Bibliografía}

1. Ecuador, entre países con tasa más alta de cáncer de tiroides. [Internet]. Ecuador: Ecuavisa; 2017 [citado 10 abril 2018]. Disponible en: http://www.ecuavisa.com/articulo/tendencias/medicina/322796-ecuador-entre-paises-

\section{$\underline{\text { tasa-mas-alta-cancer-tiroides }}$}

2. Cáncer de tiroides crece en Sudamérica y afecta a más a mujeres. [Internet]. Ecuador: El Comercio de Perú. [citado 14 de abril 2018]. Disponible en: https://elcomercio.pe/tecnologia/ciencias/cancer-tiroides-crece-sudamerica-afectamujeres-noticia-460547

3. Crecen un $200 \%$ los casos de cáncer de tiroides en Quito [Internet]. Ecuador. Redacción Médica. [citado 14 de abril 2018]. Disponible en: https://www.redaccionmedica.ec/secciones/salud-publica/crecen-un-200--los-casos-de-cncer-de-tiroides-en-quito-89559

4. González FJG. Cáncer diferenciado de tiroides. Tratamiento quirúrgico. Asignación de grupos de riesgo y análisis de supervivencia (Estudio uni y multivariante). [Tesis doctoral]. Madrid: Universidad Complutense de Madrid; 2016.

5. Jasin Amina, D Angelo Roberto, Ghiglioni Amalia BD, Boccazi D Manejo clínico del nódulo tiroideo. Guía de práctica clínica. Buenos Aires: Hospital Provincial Neuquén; 2014

6. Signos y síntomas del cáncer de tiroides [Internet]. [citado 14 de abril 2018]. Disponible en: $\quad$ https://www.cancer.org/es/cancer/cancer-de-tiroides/deteccion-diagnosticoclasificacion-por-etapas/senales-sintomas.html 


\section{Actualización sobre el cáncer de tiroides}

Vol. 2, núm. 3., (2018)

Eduardo Vera Muthre; Carmen Lazo Caicedo; Sonia Cedeño Loor; Cesar Bravo Bermeo

7. Mora J. Diagnóstico y tratamiento del carcinoma diferenciado de tiroides. Rev Esp Med Nucl Imagen Mol. 2003; 22(5):349-59. Disponible en: http://scholar.google.com/scholar?hl=en\&btnG=Search\&q=intitle:Diagnostico+y+tratami ento+del+carcinoma+diferenciado+de+tiroides \#0

8. Pitoia F, Califano I, Vázquez A, Faure E, Gauna A, Orlandi A, et al. Consenso intersocietario* sobre tratamiento y seguimiento de pacientes con cáncer diferenciado de tiroides. Rev Argent Endocrinol Metab. 2014; 51(2):85-118.

9. Medina S, Moreno F. ¿Cresta Neural o Endodermo? Conceptos divergentes sobre el origen embrionario de las células C de la tiroides. Salutem Scientia Spritius. 2017; 3(2):50-8.

10. Martín M. Estructura y función de la glándula tiroides. Rev ORL. 2016; 7(2):7-16.

11. Gil-Carcedo Sañudo E, Menéndez Argüelles ME, Vallejo Valdezate L ángel, Herrero Calvo D, Gil-Carcedo García LM. Tubérculo de Zuckerkandl. Situación, forma y dimensiones. Acta Otorrinolaringol Esp. 2012; 63(6):443-9.

12. Hernández F. Fisiología, Rendón Villa M, Mesa M. Fisiología de las glándulas tiroides y paratiroides. Barcelona: Hospital de Viladecans; 2015. Capítulo 140, IV Laringe y patología cérvico. facial; 1-18.

13. García-García C. Fisiología tiroidea. Med Int Mex. 2016; 32(5):569-75.

14. Marín M. Principios básicos de la función tiroidea. Asoc Colomb Endocrinol. 2007: 7 12.

15. Román-González A, Giraldo LR, Monsalve CA, Vélez A, Restrepo JG. Nódulo tiroideo, enfoque y manejo. Rev Med Uni de Anti. 2013; 26(2):197-206. 
Eduardo Vera Muthre; Carmen Lazo Caicedo; Sonia Cedeño Loor; Cesar Bravo Bermeo

16. Rivera S, Mesías N, Müller C, Olivares V, Capetillo M, Gabler F, Manriquez E, Vargas J. Bethesda III en resultado de PAAF de nódulos tiroideos y su correlación histopatológica. Rev Colom de Endo Diab \& Meta 2017; 4(2): 77-84.

17. Cáncer de tiroides [Internet]. [citado 18 de abril 2018]. Disponible en: https://www.seom.org/es/info-sobre-el-cancer/tiroides?showall=1

18. Hermida Pérez JA. Carcinoma papilar tiroideo con metástasis osteolíticas en una vértebra lumbar. Med Gen y Fam. 2016; 5(4):164-8. Disponible en: http://linkinghub.elsevier.com/retrieve/pii/S1889543316300044

19. ThyCa. Guía Básica del Cáncer Tiroideo. ThyCa: Thyroid Cancer Survivors' Association, $\quad$ Inc. $2013 . \quad$ Disponible en: http://www.thyca.org/download/document/416/TCBasics-Esp.pdf

20. ¿Qué es cáncer de tiroides? [Internet]. [citado 14 de abril 2018]. Disponible en: https://www.cancer.org/es/cancer/cancer-de-tiroides/acerca/que-es-cancer-de$\underline{\text { tiroides.html }}$

21. García MG, Takahashi AML, Huerta FJG, Aslan ZAT. Cáncer diferenciado de tiroides: Una antigua enfermedad con nuevos conocimientos. Gac Med Mex. 2014; 150(1):65-77.

22. Tratamiento del cáncer de tiroides según el tipo y la etapa [Internet]. [citado 14 de abril 2018]. Disponible en: https://www.cancer.org/es/cancer/cancer-de$\underline{\text { tiroides/tratamiento/por-etapa.html }}$

23. Dr. D. Herrero Calvo, Dra. E. Sánchez Terradillos DEG-CS. Cáncer de tiroides técnicas quirúrgicas sobre el tiroides. Libr virtual Form en ORL:1-22.

24. Centro Nacional de Información de Ciencias Médicas. L, Turcios Tristá SE, Velasco Mirabal M. Aplicaciones clínicas del radioyodo 131 (I131) en las enfermedades del 


\section{Actualización sobre el cáncer de tiroides}

Vol. 2, núm. 3., (2018)

Eduardo Vera Muthre; Carmen Lazo Caicedo; Sonia Cedeño Loor; Cesar Bravo Bermeo

tiroides Clinical. Rev Cuba Endocrinol. 2012; 23(3):256-63. Disponible en: http://scielo.sld.cu/scielo.php?script=sci_arttext\&pid=S1561-29532012000300011

25. Honaine L. Cancer de tiroides. Salud (i)Ciencia. 2013; 19(8):774. Disponible en: https://www.endocrino.org.co/wp-content/uploads/2015/12/Cancer_de_Tiroides.pdf

26. Sanabria, A. Chala, A. Ramírez, A. Álvarez A. Anatomía quirúrgica cervical de importancia en cirugía tiroidea. Rev Colomb Cir. 2014; 29: 50-8.

27. Navarro E. Tiroglobulina en el seguimiento del cáncer diferenciado de tiroides. Endocrinol Y Nutr [Internet]. 2005; 52(2):82-7. Disponible en: http://www.sciencedirect.com/science/article/pii/S157509220570992X\%5Cnpapers2://pu blication/uuid/FE724C31-4B58-4992-8188-A9FFA4EEDA8B 